\title{
La Corte Genaro Carrió: una nueva mirada sobre la producción de la Corte Suprema
}

\author{
"The Genaro Carrió Court": A New Look at the \\ Production of the Supreme Court
}

Magdalena Inés Álvarez

Recepción: 16/03/21

Evaluación: 20/04/21

Aceptación final: 27/04/21

\begin{abstract}
Resumen: El trabajo somete a análisis crítico tanto el argumento desplegado para justificar el enfoque tradicionalmente aplicado al estudio de la producción de la Corte Suprema, como su réplica. Presenta, a continuación, los textos que conforman La Corte Genaro Carrió. Concluye que, si bien cada texto es independiente, se enlazan entre sí a partir de una línea argumentativa común centrada en el momento histórico en el que le toca actuar al tribunal y la preocupación por sentar las bases de una cultura del argumento para una sociedad democrática.

Palabras clave: Presidencia de la Corte Suprema; inestabilidad democrática; restablecimiento del orden constitucional; cultura del argumento.
\end{abstract}

Doctora en Derecho y Ciencias Sociales (UNC), Magister en Derecho y Argumentación (UNC). Profesora de Derecho Constitucional, Facultad de Derecho, Universidad Nacional de Córdoba, Argentina. Correo electrónico: magdalenaines@gmail.com 
Magdalena Inés Álvarez

\begin{abstract}
This paper submits to critical analysis both the argument used to justify the traditional approach to the study of the Supreme Court's production and its retort. Next, the paper presents the texts that conform "The Genaro Carrió Court". It concludes that, while each text is independent, they share a common argumentative line centered on the historical moment in which the court has to act as well as the concern to lay the foundations of a culture of argument for a democratic society.
\end{abstract}

Keywords: Supreme Courts Presidency; democratic instability; constitutional order reestablishment; argumentative culture.

\title{
1. Presentación
}

Hablar de La Corte Genaro Carrió requiere ubicar la obra en el contexto de un proyecto de investigación más amplio, titulado "La Corte y sus presidencias", 1 del que constituye la primera publicación.

El proyecto - dirigido por Paula Gaido y Laura Clérico- se propone someter a estudio crítico la principal producción del tribunal, a partir de la reinstauración de la democracia (1983), utilizando como criterio de clasificación las presidencias del cuerpo.

Este enfoque - explican Gaido y Clérico- si bien es utilizado en otras prácticas jurídicas como la norteamericana, resulta novedoso en Argentina, donde la mayoría de los estudios que abordan la problemática lo hacen a partir de la relación del tribunal con quien desempeña el Poder Ejecutivo Nacional.

La razón por la que no se aplica en Argentina el enfoque seguido en el país del norte - advierten las directoras - se vincularía con la ausencia de liderazgo desarrollado por los presidentes del tribunal argentino. Gaido y Clérico cuestionan este diagnóstico por entender que se asienta en una reducción de la capacidad de "liderazgo" del presidente de la Corte a su poder de incidencia sobre la agenda del Poder Ejecutivo Nacional.

En lo que sigue, me propongo discutir tanto el argumento identificado como justificación del enfoque tradicional como la réplica efectuada por DCT 2017. 
Gaido y Clérico. Intentaré demostrar que la visión clásica que guió los estudios de la producción de la Corte Suprema de Justicia argentina, si bien pudo estar condicionada por características propias de la práctica constitucional local, estuvo determinada por el contexto de inestabilidad democrática en el que se insertó dicha práctica entre 1930 y 1983 (2). La situación cambia cuando se restablece el orden constitucional y se logra cierta continuidad democrática. Es en esta etapa en la que se inserta la investigación propuesta por Gaido y Clérico (3). Una vez zanjada esta cuestión, me concentraré en presentar La Corte Genaro Carrió. Al hacerlo sugeriré seguir una dirección de lectura ligeramente distinta a la propuesta por Clérico y Gaido, para - a continuación - presentar cada uno de los nueve textos que conforman el cuerpo principal de la obra (4). Concluiré que, si bien cada texto es independiente de los demás, se enlazan entre sí a partir de una línea argumentativa común, centrada en el momento histórico en el que le toca actuar a la Corte Carrió y la preocupación del tribunal por sentar las bases de una cultura del argumento para una sociedad democrática (5).

\section{El enfoque tradicional}

¿Por qué no han prosperado en Argentina las investigaciones sobre la Corte Suprema enfocadas en quien ejerce la presidencia del cuerpo?

La respuesta que Gaido y Clérico encuentran a esta pregunta sostiene que quienes han presidido el tribunal no desarrollaron un "liderazgo" que justifique enmarcar los estudios desde esta perspectiva.

Gaido y Clérico rechazan este diagnóstico por entender que se basa en una reducción de la capacidad de "liderazgo" del presidente de la Corte Suprema al poder de incidencia en la definición de la agenda política del Poder Ejecutivo Nacional, lo cual opaca otras aristas relevantes a ese efecto.

Tanto la respuesta como su réplica demandan un análisis más profundo. Conforme señala Vallefín (2015), los factores que han impedido que la presidencia de la Corte le imprima a esta características que permitan asociar cada etapa de la vida del tribunal con el juez que la presidió son plurales y diversos (p. 568). 
Entre dichos factores cabe investigar cómo juegan la forma en que se designa al presidente del cuerpo y el tiempo por el que permanece en el cargo.

Desde esta perspectiva, si se compara la Corte Suprema argentina con su par norteamericana se advierte que, conforme la práctica del país del norte, el Chief Justice es designado, como tal, por el Poder Ejecutivo con acuerdo del Senado por el tiempo que mantiene la investidura de juez (Vallefín, 2015, p. 571). Esto explica que solo diecisiete personas hayan presidido la Corte desde su instalación en 1789 (Federal Judicial Center), con un promedio de 13,5 años de ejercicio por cada presidencia. ${ }^{2}$

En Argentina, en cambio, el presidente del cuerpo fue nombrado por el Presidente de la Nación hasta el año 1930, fecha a partir de la cual comenzó a serlo por el propio tribunal. ${ }^{3}$ En lo que atañe a la permanencia en el cargo, la presidencia tuvo carácter vitalicio hasta el año $1947^{4}$, momento a partir del cual pasó a ser trienal (Vallefín, 2015; Benedetti, 2019b). En total treinta y una personas han encabezado la Corte Suprema argentina desde su instalación en 1863 (Santiago, 2014, p. 2029), con una duración promedio de cinco años por cada presidencia.

Si bien los factores comentados pueden haber contribuido para que el presidente del tribunal argentino no desarrolle su "liderazgo", no sirven para justificar la perspectiva de análisis de los estudios tradicionales en tanto toman como clave la relación del cuerpo con el Poder Ejecutivo Nacional.

En este marco, adquiere relevancia una tercera diferencia entre la práctica constitucional argentina y la norteamericana. Me refiero al tiempo que permanecen en el cargo los magistrados que integran la Corte. Desde esta perspec-

2 Conforme señala Vallefín (2015), la relevancia de quien ejerce la presidencia del tribunal se explica, no tanto por las funciones que le asignan las normas sino en la potencialidad extralegal con la que lo ejerce (p. 572).

3 La nueva modalidad se implementa tras el golpe de estado encabezado por el general Uriburu que destituye al presidente Hipólito Yrigoyen. Es, en consecuencia, un presidente de facto el que "prescinde" de hacer la designación que hasta entonces hacía el presidente de la Nación y reconoce a la Corte la facultad de designar a su propio presidente en ejercicio de atribuciones propias (Vallefín, 2015, p. 566)

4 Ello explica que las presidencias más extensas se den en este período. En orden decreciente: Antonio Bermejo (24 años), Roberto Repetto (14 años), José Benjamín Gorostiaga (10 años), Benjamín Paz (10 años). Francisco de las Carreras (7 años), Salvador María del Carril (7 años), Benjamín Victorica (5 años). 
tiva, si bien en ambos sistemas constitucionales la designación es efectuada con carácter vitalicio, ${ }^{5}$ si se comparan los últimos cien años, se advierte que, mientras un juez norteamericano dura en el cargo un promedio de dieciséis años, en Argentina solo lo hace por el término de siete (Castagnola, 2020, p. 52).

La desemejanza se explica porque, conforme la práctica constitucional argentina, a partir del año 1947, el recambio institucional no se produce regularmente por causas naturales - como muerte o renuncia por enfermedad o edad avanzada $-{ }^{6}$ sino que suele ser el resultado de la manipulación que ejerce el Poder Ejecutivo sobre los magistrados que integran el tribunal, con el fin de producir vacantes que permitan designar a jueces afines (Helmke, 2017; Pérez-Liñan y Castagnola, 2017; Castagnola, 2020). ${ }^{7}$

En Argentina la subordinación de la justicia al poder político remonta sus orígenes al golpe militar de 1930 cuando la Corte Suprema, sin que mediara un caso o controversia judicial, reconoció al gobierno provisional surgido de la "revolución triunfante" mediante acordada de fecha 10 de septiembre de 1930 (Cayuso y Gelli, 1988; Nino, 1992, pp. 130-131; Pellet Lastra, 2001, pp. 57-72); y se reprodujo, trece años después, cuando la legitimidad de un nuevo gobierno de facto fue reconocida por acordada emitida el 9 de junio de 1943 (Pellet Lastra, 2001, pp. 82-94; Castagnola, 2020, pp. 64-65).

Restablecido el orden constitucional, bajo la presidencia de Juan Domingo Perón, se produjo la primera destitución masiva de miembros del tribunal cuando, cuatro de sus cinco ministros y el Procurador General de la Nación, fueron sometidos a juicio político. ${ }^{8}$ Como resultado, fueron removidos el Procurador General y tres de los cuatro jueces enjuiciados (Pellet Lastra, 2001, p. 133; Abásolo, 2013, p. 483).

5 Constitución Estados Unidos de América, artículo III, sección 1. Constitución Nacional, artículo 110 .

6 El recambio natural sí operó, en cambio, entre los años 1863 y 1947 (Santiago, 2013, p. 6).

7 Conforme Castagnola (2020) en Argentina, entre los años 1930 y 2014, de un total de 86 jueces que se han desempeñado en el cargo, 52 (60\%) fueron inducidos al retiro. La mayoría de esos retiros forzados tuvieron lugar después de un cambio de régimen (62\%), mientras que el resto devino como resultado de procesos de juicios políticos (19\%), retiros inducidos amigables (11\%), y como manipulación política de la justicia (8 \%) (p. 57-58).

8 El pedido de juicio político, presentado por el diputado Rodolfo Decker, solo excluía del proceso de remoción al juez Tomás Darío Casares. En cambio, incluía a Roberto Repetto aun cuando ya había renunciado al cargo y la renuncia le había sido aceptada por el Poder 
Mas el juicio político sustanciado en el año 1947 no constituyó la única purga que sufrió la Corte Suprema argentina. A partir de este hecho la posibilidad de designar más de la mitad de los miembros del cuerpo dentro de un mismo gobierno, como resultado de retiros inducidos ${ }^{9}$, se reprodujo en ocho oportunidades más: $1955,1960,{ }^{10} 1966,1973,1976,1983,1990$ y 2003 2006 (Castagnola, 2020, pp. 61; García Mansilla, 2020; Pellet Lastra, 2001).

La doctrina especializada advierte que el recambio judicial de los tribunales superiores puede ser explicado aplicando el concepto de "eventos en serie". Estos son "respuestas recurrentes entre los actores políticos para relocalizar el poder, produciendo interrupciones cíclicas en el status quo" (Pérez-Liñan y Castagnola, 2017, p. 54).

Explican Pérez-Liñan y Castagnola (2017) que las purgas reiteradas a las que se ven sometidos los tribunales superiores revelan una batalla entre quienes detentan el poder para asegurar el dominio político sobre el Poder Judicial. En esta batalla se disputan bienes tangibles - los asientos del tribunal - y cada respuesta produce una redistribución del poder para ejercer la revisión judicial (p. 55).

La reiteración de los eventos se explica por la articulación de dos lógicas diferentes: la primera sostiene que existen factores latentes de riesgo constante que generan la posibilidad de que surjan estos eventos. ${ }^{11} \mathrm{La}$ segunda destaca que el riesgo aumenta en forma creciente a medida que se reiteran los episodios. ${ }^{12}$ De allí que cuantos más episodios se concatenan,

Ejecutivo. El Senado, finalmente, resolvió excluir Roberto Repetto por entender que, al habérsele aceptado la renuncia con anterioridad al inicio del procedimiento, ya no estaba sujeto a la jurisdicción del tribunal (Pellet Lastra, 2001, p. 112; Abásolo, 2013, p. 483).

9 Las tácticas a la que recurren los poderes políticos para manipular la integración de la Corte van desde el juicio político, la remoción por decreto de gobiernos de facto, la designación de nuevos magistrados cuando se restablece el orden constitucional, pasando por la ampliación del número de miembros del tribunal, hasta estrategias informales, como amenazas y persecución política (Castagnola, 2020, p. 60).

${ }^{10}$ En 1958 la estrategia incluyó la confirmación de dos de los jueces designados por la Revolución Libertadora y la ampliación del número de ministros que conforman el tribunal por Ley 15.271 sancionada en el año 1960 (Pellet Lastra, 2001, pp. 209-211 y 233-236).

${ }^{11}$ Como la inestabilidad institucional recurrente o las reformas reiteradas de la Constitución.

${ }^{12}$ La razón de que ello ocurra se relaciona con la pérdida de legitimidad del tribunal que produce cada purga y la conformación de tribunales partidistas, todo lo cual incentiva volver a 
mayores son las probabilidades de experimentar el mismo evento nuevamente (Pérez-Liñan y Castagnola, 2017, pp. 57-58).

Desde esta perspectiva, el hecho de que cada gobierno que asume se sienta habilitado para recurrir a estrategias de manipulación para contar con una Corte amigable, constituye un factor de peso que incide en los investigadores al momento de decidir el enfoque a partir del cual estudiar el funcionamiento y producción del máximo tribunal.

\section{La pertinencia del nuevo ángulo de análisis}

Cabe observar que, conforme explican Pérez-Liñan y Castagnola (2017), la cíclica reproducción del recambio judicial no constituye un legado inevitable ya que el riesgo que enfrenta el tribunal de sufrir manipulaciones cambia a partir de la modificación del contexto político (p. 68).

Estudios recientes afirman que, a partir del año 1983, cuando Argentina comienza a actuar conforme a reglas de juego democráticas, estas prácticas sufren una desaceleración ${ }^{13}$ debido a que el costo político y social asociado con la manipulación pasa a ser más alto que el beneficio obtenido por los poderes políticos al instrumentarla (Pérez-Liñan y Castagnola, 2017, pp. 68-69. Castagnola, 2020).

Esto justifica que, a partir de 1983, las investigaciones basadas en la relación de subordinación judicial al Ejecutivo reduzcan su atractivo y adquieran relevancia otras miradas.

La investigación dirigida por Gaido y Clérico se inserta en esta concepción en tanto toma como punto de partida el inicio de la recuperación democrática, momento a partir del cual —afirman— les interesa producir

alterar su integración cada vez que cambia la titularidad del Poder Ejecutivo (Pérez-Liñan y Castagnola, 2017, p. 68).

${ }^{13}$ Esto no significa que la manipulación desaparezca por completo. En Argentina, una vez reinstaurada la democracia, se presentaron dos casos exitosos de manipulación (Menem, 1990 y Kirchner, 2003-2006). A ello se agrega la amenaza de Eduardo Duhalde de someter a juicio político a los integrantes del tribunal (2002) que, si bien no tuvo éxito, incidió en la subordinación del tribunal al Poder Ejecutivo. 
herramientas que, desde la teoría, sirvan para la construcción de una mejor práctica jurídica (Gaido y Clérico, 2019, p. 16).

La propuesta resulta novedosa no solo porque estructura el estudio de la producción del tribunal a partir de quien ejerce su presidencia, sino también porque atribuye especial relevancia a la reconstrucción del contexto político-social en el que le toca actuar a cada Corte y amplía la mirada para abarcar tanto la producción jurisprudencial como las acordadas y resoluciones emitidas bajo cada presidencia.

Este último material — señalan Gaido y Clérico— constituye un ámbito de estudio no explorado en forma sistemática, cuya revisión es de utilidad para identificar rivalidades internas; tensiones hacia adentro del Poder Judicial; relaciones entre poderes; vínculos con organismos internacionales, medios de comunicación e - incluso - con la comunidad. De esta manera, se busca identificar todas aquellas aristas que confieren relevancia a la figura del presidente del tribunal que quedaban opacadas con el enfoque tradicional.

Finalmente, cabe destacar que el abordaje se efectúa a partir de una mirada interdisciplinaria y se instrumenta como trabajo colectivo mediante el diálogo constante entre autores, editores y directores.

\section{El contenido de La Corte Genaro Carrió}

La Corte Genaro Carrió comienza con una presentación del proyecto de investigación "La Corte y sus presidencias" que expone y justifica el enfoque que Clérico y Gaido dan a la investigación.

En dicha presentación, sin embargo, no se abordan aspectos que pueden resultar relevantes para apreciar esta mirada. Esto no significa que Clérico y Gaido no adviertan la necesidad de trabajar estas cuestiones; mas difieren su tratamiento.

Con el fin de orientar al lector, sugiero anticipar la lectura del trabajo elaborado por Miguel A. Benedetti, titulado "Los otros presidentes: designación y duración de la presidencia de la Corte Suprema Argentina (18632018)", que se publica al final de la obra bajo el título "Anexo".

Allí encontrarán una interesante y medulosa reflexión, acerca de los sistemas de designación y de duración de los presidentes de la Corte 
Suprema en el cargo, ensayados desde mediados del siglo XIX hasta la actualidad; y la influencia que estos sistemas tienen en la importancia que fueron adquiriendo los presidentes del alto tribunal.

Como explica el autor, si bien en el pasado el cargo de presidente no se correspondía con una gran carga de trabajo, esto cambia a medida que se acrecienta el rol que desempeña la propia Corte quien, por medio de acordadas, va atribuyendo al presidente distintas tareas que se suman a las que le asigna el legislador ordinario.

De esta manera, concluye Benedetti, el rol de primus inter pares que tradicionalmente desempeñaba el presidente del tribunal se va transformando hasta convertirse en primus sobre pares.

Dado que La Corte Genaro Carrió no se estructura en capítulos, en lo que sigue presentaré los textos que conforman el cuerpo principal de la obra identificándolos por su título y autor.

\section{1. "La Corte Genaro Carrió: hacia una cultura del argumento para una sociedad democrática”. Laura Clérico y Paula Gaido}

El primer texto presenta la mirada que atraviesa toda la obra: me refiero al análisis de la producción de la Corte Carrió en el contexto del restablecimiento de la democracia, que es considerado por Clérico y Gaido como ineludible ángulo de lectura.

El trabajo expone las razones que tornan insoslayable terminar con la Corte de la dictadura y proceder a la integración de un nuevo tribunal comprometido a enfrentar el pasado reciente.

Destaca también la relevancia que atribuye la Corte Carrió a los derechos y garantías, cuyo control desarrolla no solo indagando si el acto que restringe derechos respeta el principio de legalidad, sino dando especial trascendencia al análisis de si existen razones que justifiquen su eventual restricción.

Estas dos miradas explican la selección de los "focos jurisprudenciales" que se analizan en la obra: los dos primeros (justificación de la justicia civil para juzgar a los militares y ruptura con la doctrina de facto) responden a la urgencia de dar respuestas al pasado dictatorial, mientras que los tres res- 
tantes (“Arenzón", "Poncetti de Balbín” y "Fiorentino") reflejan la clave argumentativa que la Corte quiso instalar en materia de derechos y garantías.

Para Clérico y Gaido, la Corte Carrió inaugura un período en el que se sienta una "jurisprudencia de oro" en materia de derechos humanos y garantías; y las bases para la consolidación del Estado de derecho y la democracia.

Bajo la presidencia de Carrió — afirman — la agenda de la Corte plasmó la transformación de una cultura autoritaria en otra que exige a los poderes del Estado "dar razones", "justificar" sus actos. Esta mirada se extendió, incluso, hacia el interior del propio tribunal, ámbito en el que Carrió imaginó una Corte que habla "con una sola voz y sin estridencias".

En definitiva - concluyen las autoras- el diálogo, la deliberación y la cultura del argumento son los legados que dejó la Corte Carrió.

\section{2. "El Poder Judicial en los primeros años de la democracia: prácticas cotidianas y rituales de cambio". Leticia Barrera}

En este texto Leticia Barrera se aproxima al campo jurídico desde una mirada que asume el derecho, no como un sistema cerrado y autónomo, sino como un campo en el que se expresa lo social y en el que confluyen intereses políticos, ideologías y moralidades.

Ello explica que proponga una interpretación contextualizada que se ocupa de dos aspectos del campo jurídico: por un lado, de los efectos simbólicos del discurso, los actos y las prácticas en relación a los reclamos de justicia y, por otro, de la política del nuevo gobierno democrático con relación al Poder Judicial.

La autora se detiene en el análisis de las formas jurídicas en las que se plasma la renovación de la Corte. Muestra cómo, despojadas de contexto, presentan los hechos en un marco de continuidad y regularidad. Esta lectura cambia cuando se los contextualiza y sitúan en el momento político determinado y crítico en el que se dan: el retorno al sistema democrático luego de siete años de dictadura.

En lo que atañe a la composición del Poder Judicial, explica Barrera, no estaba en la mente de Alfonsín dinamitarlo, pero tampoco mantener la integración que venía de la dictadura. Ello motivó la puesta en comisión 
de todos los jueces y el requerimiento del acuerdo del Senado para continuar en el cargo. Como resultado, fueron confirmados la gran mayoría de los magistrados, excepto aquellos impugnados por causas de derechos humanos cuyos pliegos quedaron fuera de discusión al no ser propuestos por el Ejecutivo.

De allí que - concluye Barrera-durante los primeros años de la transición democrática se mantuvo estable la dinámica del funcionamiento del Poder Judicial, apoyada en la relación establecida entre agentes judiciales que trasciende a los gobiernos. De esta manera la función jurisdiccional, entendida como actividad técnica, se proyectó como continua y contrastó con la decisión del Poder Ejecutivo de darle un sentido marcadamente político a la designación de los miembros de la Corte.

La decisión del gobierno de tomar distancia del régimen precedente, por otra parte, marcó fuertemente el contexto político en el que se desempeñaba el Poder Judicial. Esta decisión se plasmó en actos con fuerte sentido simbólico, como la sanción de la Ley 23040, que declaró insanablemente nula la autoamnistía y negó su fuerza obligatoria desde el nacimiento; la reforma de los tribunales comprometidos con el juzgamiento de violaciones de derechos humanos; $\mathrm{y}$-muy especialmente- el juicio a los ex comandantes de la junta militar.

De esta manera - concluye la autora - el carácter extraordinario del ritual de cambio entra en tensión con la continuidad de la práctica judicial. Mas, lejos de suprimirse recíprocamente, ambos coexisten en relación dialéctica, alimentando una dinámica propia de funcionamiento del Poder Judicial.

\section{3. “Genaro R. Carrió”. Alberto Garay}

Alberto Garay asume la tarea de proporcionar al lector una semblanza aproximada de Genaro Carrió.

Nacido en Concordia, provincia de Entre Ríos - explica Garay-, Carrió cursó sus estudios universitarios en la ciudad de La Plata, donde se vio deslumbrado por las enseñanzas de Carlos Cossio y Ambrosio Gioja. Una vez recibido, entrelazó el ejercicio profesional con la actividad académica, siendo fundamental las estancias que pasó en Estados Unidos (1954- 
1956) y en Oxford (1964 y 1968-1969), en donde trabajó bajo la supervisión del filósofo Herbert L. A. Hart.

Para Garay, en el legado de Carrió, tan importantes son las obras de su autoría como las numerosas traducciones que hizo de autores de habla inglesa que, por primera vez, podían ser leídos en Argentina por un público al que - en la década del 80- le era difícil conseguir bibliografía actualizada.

En lo que atañe a la defensa de los derechos humanos, Garay destaca el paso de Carrió por la Comisión Interamericana de Derechos Humanos (1972) - cargo al que renunció por la inacción de la Asamblea General de la OEA frente al caso chileno- ${ }^{14} \mathrm{y}$, particularmente, el patrocinio de habeas corpus de detenidos por el régimen militar argentino.

Restablecida la democracia, Raúl Alfonsín lo propuso como ministro de la Corte, siendo luego designado presidente del cuerpo por el voto de los jueces que integraban el tribunal. Mas la sobrecarga de tareas, la falta de acuerdo respecto de la forma en que debe trabajar un cuerpo colegiado y las enemistades internas hicieron que la permanencia de Carrió en el tribunal fuera breve.

El afectuoso retrato que pinta Garay de Genaro Carrió tiene la virtud no solo de acercar al lector a la imagen pública de quien califica como una de las mentes jurídicas argentinas más brillantes, si no que - a la vezdevela aspectos de su vida privada - por ejemplo, el vínculo que lo unió a su familia—, sus estados de ánimo - feliz en Oxford, extremadamente triste a su regreso, abatido y frustrado en su paso por la Corte- e, incluso, de su salud que se apagaba.

La Corte, explica Garay, no le brindó a Carrió la armonía que necesitaba para desarrollar sus capacidades. Renunció el 17 de abril de 1985, a los 63 años de edad. A partir de entonces — concluye Garay (2019) — Genaro Carrió “... nunca volvería a ser el de antes” (p. 77).

${ }^{14}$ País al que la Comisión Interamericana concurrió para investigar denuncias por violaciones masivas de derechos humanos. 


\section{4. "Una reconstrucción (no jurisdiccional) de la Corte presidida por Genaro Carrió. Tras los rastros de las acor- dadas y resoluciones más significativas". Miguel Benedetti}

En cuarto lugar, se agrega un novedoso estudio en el que se analiza la llamada "actividad no jurisdiccional" del tribunal, expresión con la que se busca aprehender "una gama heterogénea y sub explorada de facultades constitucionales del tribunal como cabeza del Poder Judicial de la Nación que se ejercen por fuera de la actividad propiamente jurisdiccional que se manifiesta por medio de sentencias" (Benedetti, 2019a, p. 79).

Esta línea, precisa Benedetti, asume que las acordadas y resoluciones no solo abordan cuestiones burocráticas o de organización interna sino que se emplean como medio de intervención pública y expresión política del tribunal frente a la sociedad, los poderes políticos y el propio Poder Judicial.

En orden a la forma de abordar este material, el autor precisa que no es su intención efectuar un inventario de todas las acordadas y resoluciones investigadas. Se decanta, en cambio, por una lectura panorámica que - aclara- permite detectar más conexiones de las que se podría esperar.

Este análisis toma particularmente en cuenta la transición democrática de esos años - calificada por Nino como "única” y sui géneris (Benedetti, 2019a, p. 89) - como el gran marco contextual en el que se dan, en forma simultánea, otros procesos de transición más limitados, pero que también tienen su origen en el pasado dictatorial e impactan en las actuaciones no jurisdiccionales de la Corte Carrió.

A la hora de categorizar la problemática que debía abordar la Corte, el autor considera insuficiente la dicotomía "pasado-presente" y se decanta por la distinción entre problemas "residuales" y "emergentes", utilizando las expresiones en el sentido propuesto por Williams (Benedetti, 2019 a, p. 91). Concluye que la Corte Carrió recurre a una tradición selectiva, mediante la inclusión y exclusión de prácticas que combinan precedentes de determinados períodos en los que el Poder Judicial recupera su dignidad y jerarquía, con innovaciones en múltiples temáticas, algunas de ellas bastante audaces para el momento que se atravesaba.

Finalmente, la aplicación de la hipótesis de lectura propuesta al material seleccionado lleva al autor a señalar que la Corte hizo un manejo prudente - 
pero a la vez activo y alerta - de las facultades constitucionales como cabeza del Poder Judicial en relación a los otros poderes de la democracia recuperada; a la vez que dio respuesta a problemas "residuales" - como la cuestión de la "Morgue Judicial" o la ratificación y revisión de la situación de funcionarios judiciales designados durante el gobierno de facto- $\mathrm{y}$ atendió los asuntos "emergentes" - como la reorganización interna de la propia Corte; la adopción de sistema de concursos públicos para la designación de funcionarios judiciales que requieren título habilitante; el control reglamentario sobre la conducta de jueces y funcionarios; así como también las innovaciones (y reacciones) frente a distintas situaciones del personal judicial一.

De esta manera, concluye el autor, la polifacética actuación no jurisdiccional muestra cómo la Corte Carrió, a pesar de ser una de las más breves en la historia del tribunal, colaboró en la empresa de restablecer las instituciones del Estado de derecho.

\section{5. "Justicia militar, justicia federal". Leonardo Filippini}

A continuación, la obra presenta el trabajo titulado "Justicia militar, justicia federal" en el que se aborda el primer "foco jurisprudencial" seleccionado por las editoras. Me refiero a la ubicación de la jurisdicción castrense en democracia.

Recuerda Filippini que, en diciembre de 1983, el Poder Ejecutivo convocó a sesiones extraordinarias para tratar un grupo de leyes entre las que se encontraba la reforma del Código de Justicia Militar. El proyecto original enviado por el Poder Ejecutivo al Congreso de la Nación reconocía la jurisdicción castrense para juzgar los hechos cometidos en el pasado, a la vez que atribuía a las Cámaras Federales de Apelaciones amplios poderes de revisión de las decisiones de aquella jurisdicción.

El Congreso de la Nación, al debatir el proyecto, le introdujo modificaciones relevantes, que - en palabras de Nino- "frustraba la estrategia de Alfonsín" quien, sin embargo, decidió no intervenir y aceptar la suerte del proyecto (Filippini, 2019, p. 128. Nino, 2015, p. 147).)

En definitiva, en lo que aquí interesa, la ley sancionada atribuyó a la jurisdicción castrense el conocimiento de los delitos cometidos con ante- 
rioridad a la vigencia de la ley, con un recurso ante las Cámaras Federales de Apelaciones. En caso de demora injustificada o negligencia en la tramitación del juicio, la Cámara podía también asumir el conocimiento del proceso, cualquiera fuera el estado en que se encontraran los autos.

Frente a la nueva ley, explica Filippini, la Corte Carrió se centró en definir el lugar de la jurisdicción castrense en democracia. Así: en "Fiscalía Nacional de Investigaciones Administrativas" hizo una lectura estricta de lo que se entiende por "acto de servicio"; en "Gauna” limitó la intervención de la jurisdicción militar respecto de civiles y falló en "Giusti” a favor del imputado, a pesar de la interposición extemporánea del recurso, por considerar que el Consejo Supremo Militar había violentado la garantía de la reformatio in peius.

En lo que atañe a la adopción de mecanismos específicos para abordar crímenes de la dictadura, al resolver los casos "Competencia 40" y "Bignone”, la Corte contribuyó a llevar adelante el juicio a la junta militar al aceptar - en un primer momento- que sean realizados por tribunales militares conforme pretendía el poder político. Mas tarde, en "Videla”, avaló también la decisión del Congreso de asignarle a las cámaras federales la potestad de avocarse frente a la demora injustificada o negligencia en la tramitación del tribunal castrense.

El último caso, destaca Filippini, resulta particularmente relevante porque en él la Corte explicó el enfoque que asume sobre la interpretación constitucional. Al hacerlo, dio particular importancia al "contexto" que —explicó el tribunal- "no está integrado por palabras sino de 'historia y finalidades"'. Desde esta mirada — agrega - cabe reconocer que los militares pueden invocar como jueces naturales a los tribunales castrenses para los delitos cuya modalidad autorice a incluirlos en la competencia castrense (Filippini, 2019, pp. 152-153).

Mas, precisa el autor, la Corte afirmó abrevar en su propia línea interpretativa para justificar la constitucionalidad de las restricciones impuestas por el artículo 10 de la Ley 23049 a las atribuciones conferidas al Consejo Supremo de las Fuerzas Armadas.

Para Filippini, la transición democrática marcó el tono dramático del grupo de decisiones sobre jurisdicción militar. Los jueces, señala el autor, estaban persuadidos del contexto en el que se toman las decisiones y acom- 
pañaron a la administración en el experimento de condenar el pasado, pero sin poner en cuestión al futuro.

En este marco, concluye el autor, la Corte produjo decisiones originales que buscaban legitimar en líneas argumentales ya desarrolladas por el tribunal en torno a principios basales de la Constitución. De esta manera, la Corte colaboró en romper con el pasado inmediato, pero sobre la base de referencias tangibles a principios anclados en la memoria colectiva de libertades constitucionales, plegándose a la idea de la época según la cual se había extraviado el camino de civilidad al que se podría retornar.

\section{6. "Normas de facto/normas de iure: la influencia filosófica en la Corte Carrió”. Gustavo Beade, Camila Petrone y María de los Ángeles Ramallo}

El segundo "foco jurisprudencial" se centra en la validez de las decisiones emanadas de un gobierno de facto y es abordado por Gustavo Beade, Camila Petrone y María de los Ángeles Ramallo.

La problemática presentaba especial relevancia en 1983, no solo por la extensión de la interrupción del orden constitucional sino, por la decisión de la junta militar de otorgar una amplia autoamnistía. Esta ley constituía un obstáculo para los juicios de abusos a los derechos humanos toda vez que, aun cuando se la derogara, ello no impedía su aplicación en virtud del artículo 2 del Código Penal, que garantizaba a los imputados el beneficio de la ley más favorable (Nino, 2015, p. 134).

Entre la convalidación plena y el desconocimiento global de la legislación de facto un grupo de académicos comprometidos con la transición democrática propuso una tercera alternativa: otorgarle una validez precaria, fundada en la necesidad y no en una presunción de justicia que emana del procedimiento de origen democrático.

Conforme esta alternativa, las normas de facto gozan de una validez prima facie que es determinada por consideraciones de paz y seguridad. De allí que sean obligatorias solo hasta que sea evaluada su adecuación axiológica. Los jueces tienen la obligación de valorar el contenido de las normas de facto. Dada la precariedad de estas normas, además, ellas no 
generan derechos adquiridos antes que se haya corroborado su legitimidad (Nino, 1983; Nino, 1985).

Reinstaurada la democracia, la Corte decidió seguir la línea propuesta, abandonando la llamada "doctrina de facto" sostenida desde el golpe de 1930. Lo ocasión se presentó el 14 de febrero de 1984 cuando el tribunal resolvió el caso "Aramayo". A este fallo le siguieron "Dufourq", "Bosch", "Gary", "Buzzetti Thomson" y "Brieva”.

Beade, Petrone y Ramallo advierten que los comentaristas suelen sostener que las decisiones adoptadas en todos estos casos constituyen un grupo uniforme en los que el tribunal construye una doctrina sobre el problema. Los autores no están persuadidos por esta afirmación. Entienden, en cambio, que se trata de problemas distintos que el tribunal presenta, en forma insatisfactoria, como uno solo. ${ }^{15}$

En "Aramayo" el actor, que había sido designado como juez por el gobierno democrático anterior y removido por el gobierno de facto, solicitó que se lo restablezca en el cargo. El planteo fue rechazado por la Corte. Para así resolver, el tribunal afirmó que la restitución del orden constitucional requiere que los poderes del Estado nacional o de las provincias, en su caso, ratifiquen o desechen explícita o implícitamente los actos del gobierno de facto. Dejó, de esta manera, de lado explícitamente la validez de las normas dictadas durante la dictadura militar y las condicionó a la decisión que tome el Estado.

Como observan Beade, Petrone y Ramallo, el criterio transcripto no justifica por sí solo la decisión a la que llegó el tribunal en "Aramayo". Ello toda vez que el reclamante había sido designado por un gobierno democrático y removido por un gobierno de facto. De allí que, si las normas de facto no son normas válidas, una decisión apoyada en este argumento le habría permitido a Aramayo retornar al cargo. Esto los lleva a buscar otros

15 Destacan que, mientras el primer caso trata sobre un juez designado por un gobierno de iure que es destituido por el gobierno militar, los restantes se refieren a: a) jueces y/o funcionarios judiciales designados por el gobierno democrático ratificados por la dictadura que luego no son confirmados al reinstaurarse la democracia ("Bosch" y "Brieva"); b) jueces y/o funcionarios judiciales directamente designados por el gobierno de facto que no son confirmados por el gobierno de iure (“Dufourq", "Buzzetti Thomson”, “Gary”). 
fundamentos que - afirman - son los que inclinan la balanza de razones en contra de la pretensión del actor.

En "Dufourq" y "Buzzetti Thomson" la Corte agregó que un gobierno de facto puede sancionar normas válidas para el futuro, pero no integrar poderes de iure, que solo puede ser hecho conforme a la directiva del art. 86 inc. 5 de la CN (texto 1853-60). Este argumento justifica que los jueces designados durante la dictadura no gocen de la garantía de la inamovilidad.

En "Bosch" y "Brieva" - siguiendo el presente sentado en "Sagasta" (1958) - precisó que también carecen de la garantía de la inamovilidad aquellos jueces que, si bien fueron nombrados con anterioridad al gobierno de facto, fueron puestos "en comisión" y confirmados por este.

Si bien Beade, Petrone y Ramallo consideran que la doctrina sentada por la Corte Carrió es correcta, cuestionan el alcance que se les otorga. En tal sentido están de acuerdo con que un gobierno constitucional tiene que poder designar a sus propios jueces. Mas - afirman - el argumento no funciona en los casos "Aramayo", "Bosch" y "Brieva".

En este marco, se preguntan si es posible identificar un fundamento común a la resolución de estos casos que justifique las decisiones adoptadas. Lo encuentran a partir de algunas nociones de ética normativa. Sostienen que la Corte - particularmente en "Aramayo" - construyó un concepto de seguridad jurídica basado en principios utilitaristas. Dicho concepto demanda preservar la regularidad de la transición democrática a la vez que evitar la proliferación de reclamos que podría haber dado lugar resolver el caso de manera diferente. Esta construcción - agregan - es la que permite que la seguridad jurídica siempre se imponga en la ponderación de los derechos alegados por los demandantes.

Mas allá que se pueda estar de acuerdo con la naturaleza utilitarista del argumento empleado por el tribunal para justificar la decisión adoptada en "Aramayo", su extensión a "Bosch" y "Brieva" resulta — al menosdiscutible. Ello pues estos casos presentan propiedades relevantes que los asemejan a "Dufourq" y "Buzzetti Thomson", a la vez que los distinguen de "Aramayo". Si bien el título de "Bosch" y "Brieva” provenía originalmente de un gobierno de iure, la posterior puesta "en comisión" por el gobierno de facto privó al cargo de la garantía de la inamovilidad conferida por el art. 96 de la Constitución Nacional (texto 1853-60). La nueva designación 
por el Ejecutivo de facto, al no ser efectuada conforme lo reglado por el art. 86 inc. 5 de la Constitución Nacional (texto 1853-60), no purgó el vicio. Tampoco lo hizo el artículo 10 del Estatuto para el Proceso de Reorganización Nacional cuya validez, a lo sumo, alcanza al período de la dictadura.

Esto no significa desconocer que de un argumento por analogía no deriva certeza. La conclusión, en consecuencia, no pretende cerrar el debate sino reabrirlo hacia un nuevo intercambio de razones que permita evaluar la fuerza de los argumentos en pugna.

\section{7. "Razonabilidad, igualdad y educación en escena: el caso 'Arenzón”'. Liliana Ronconi, Victoria Fiorotto y Martina Galli}

En lo que sigue la obra se concentra en tres sentencias de la Corte Carrió especialmente seleccionadas por las editoras por considerar que constituyen ejemplos paradigmáticos de la clave argumentativa que el tribunal quiso implementar en materia de derechos y garantías.

El primer trabajo, titulado la "Razonabilidad, igualdad y educación en escena: el caso 'Arenzón”', aborda la sentencia dictada por el tribunal en el caso identificado por Petracchi (1988) como "el caso del profesor petiso".

En él, la Corte trató una acción de amparo interpuesta en contra de la decisión de la Dirección de Sanidad Escolar de negar el certificado de aptitud psicofísica para ingresar al profesorado de matemática y astronomía a una persona que no reunía el requisito de altura $-1,60 \mathrm{~m}-$ requerido por la resolución 957/81-.

Ronconi, Fiorotto y Galli observan que, el contexto político y social en el que se dicta la resolución, apareció en forma clara en el voto concurrente firmado por los ministros Petracchi y Belluscio que afirmó que la Corte no puede prescindir del hecho de que "la exigencia ha sido establecida por una autoridad de facto, lo que exige una revisión judicial honda y puntual". Advierten que el voto comentado tomó en cuenta también el contexto en el que se dictó la sentencia, en cuanto refiere a la incompatibilidad del requisito de la estatura mínima con "la necesidad de cimentar una sociedad democrática e inteligente" y que esta exigencia "mancha el ordenamiento jurídico argentino con valores éticos sustancialmente anacrónicos”. 
Las autoras precisan que la Corte aplicó un método interpretativo que se relaciona con la visión sobre el papel de los jueces defendida por Ely (1997). Para dicho autor el control de constitucionalidad se ejerce para proteger el proceso democrático. Siguiendo este razonamiento, los jueces solo intervienen cuando las leyes se entrometen sospechosamente en derechos asociados al proceso democrático y atenten contra las minorías.

El fallo sostuvo que la negativa de extender el certificado de aptitud psico-física no guardaba relación razonable con el objetivo de estudiar el profesorado de matemática y astronomía; advirtió que la necesidad de la medida no pudo ser probada por el Ministerio y destacó que las circunstancias de que el Ministerio obrase en ejercicio de facultades discrecionales, no justificaba una conducta arbitraria, ya que es la razonabilidad con que se ejercen esas facultades lo que da validez a los actos de gobierno.

En orden al impacto de esta sentencia en decisiones futuras, las autoras proponen tres ejes que surgen de tres reglas implícitas que la Corte elaboró o dejó latente en su argumentación. Dichos ejes son:

- El ejercicio de facultades discrecionales de la Administración no puede ser arbitrario: toda medida debe guardar un vinculo de razonabilidad comprobable entre el medio elegido y el fin perseguido.

- La educación que sostiene una comunidad democrática es aquella en la que se brindan razones públicas y razonables en el proceso de enseñanza-aprendizaje.

- Toda distinción basada en la apariencia física o similar exige brindar razones objetivas y razonables para ser válida.

Esta última regla — afirman Ronconi, Fiorotto y Galli- se explica porque, si bien el fallo no trabajó en forma expresa el principio de igualdad, subyace a la argumentación desarrollada una concepción de igualdad que no se limitó a exigir un trato idéntico entre quienes caen dentro de la clasificación que realiza la norma y trato desigual respecto de quienes se encuentran fuera de esa clasificación, si no que empezó a preguntarse sobre la "razonabilidad de la distinción". Comienza, en consecuencia, a exigir razones que hablen a favor o en contra del criterio utilizado por el legislador para clasificar. 
Estas reglas son retomadas, sostenidas y reelaboradas a lo largo de las siguientes composiciones del tribunal, tal como lo demuestran Ronconi, Fiorotto y Galli en el trabajo que se comenta.

\section{8. "El caso 'Ponzetti de Balbín': el choque entre la libertad de expresión y el derecho a la intimidad”. Hernán Gullco}

A continuación, la obra presenta el trabajo titulado "El caso 'Ponzetti de Balbín': el choque entre la libertad de expresión y el derecho a la intimidad”.

El caso, originado en la demanda de daños y perjuicios iniciada por la esposa e hijo del Ricardo Balbín contra la Editorial Atlántida por la publicación de una fotografía del político radical cuando agonizaba en terapia intensiva, llegó al Corte vía un recurso extraordinario federal interpuesto por la parte demandada.

Para Gullco la importancia de la sentencia radica en que configura el primer análisis moderno efectuado por el tribunal respecto de la tensión entre libertad de expresión y el derecho a intimidad, entendidos ambos como derechos que emergen del texto constitucional (art. 14 y $19 \mathrm{CN}$ ).

Cabe prestar atención a un dato estructural de la decisión: se trata de una sentencia que, si bien es unánime en cuanto a lo que resuelve, no lo es en sus fundamentos toda vez que está conformada por tres votos concurrentes, cada uno de los cuales desarrolla su propia cadena argumentativa. Determinar qué argumentos tienen mayoría a fin de precisar la doctrina que emerge del fallo es una tarea que asume quien comenta la decisión.

El problema a resolver es identificado por Gullco a partir del voto suscripto por los ministros Carrió y Fayt, que destaca que, en el caso, no está en juego el derecho de publicar las ideas por la prensa sin censura previa (art. $14 \mathrm{CN}$ ) sino los límites al derecho a la información en relación directa con el derecho a la intimidad (art. 19 CN). En consecuencia, observa el autor, no se plantea una "restricción previa" al ejercicio de la libertad de expresión sino de una "restricción ulterior" (art. 13.2 Convención Americana de Derechos Humanos).

El autor resalta el reconocimiento que - al momento de resolver el caso- tenía la libertad de expresión en la jurisprudencia del tribunal, reco- 
nocimiento que encuentra fundamento en la relación estrecha que guarda este derecho con la democracia.

Para justificar esta afirmación, Gullco cita el precedente "Abal" (1960) en el que la Corte afirmó:

(...) entre las libertades que la Constitución Nacional consagra, la de prensa es una de las que poseen mayor entidad al extremo de que sin su debido resguardo existiría tan solo una democracia desmedrada o puramente nominal" ... "está claro que la Constitución al legislar sobre libertad de prensa, protege fundamentalmente su propia esencia democrática contra toda posible desviación tiránica.

Mas, la decisión en "Ponzetti de Balbín" se decantó por dar primacía en el caso- al derecho a la intimidad respecto de la libertad de expresión.

Las razones que sustentaron la decisión son sintetizadas por Gullco en la siguiente forma:

- La libertad de expresión es un aspecto central de un estado democrático.

- El derecho a la intimidad, que también tiene fundamento constitucional, requiere una fuerte tutela judicial.

- La actuación pública o privada de los "personajes públicos" puede divulgarse en lo que se relacione con la actividad que les confiere prestigio o notoriedad siempre que lo justifique el interés general.

- El avance sobre la intimidad no autoriza a dañar la imagen pública o el honor de estas personas y menos sostener que no tienen un sector de vida privada protegida de toda intromisión.

- Dado que la difusión de las fotografías no está justificada en un interés general, corresponde concluir que la publicación de la revista demandada no se encuentra tutelada por la libertad de expresión.

La doctrina suele destacar también otros argumentos desarrollados por la sentencia; ${ }^{16}$ mas, al carecer de apoyo mayoritario, estos argumentos no

${ }^{16}$ Por ejemplo, el considerando 8 del voto firmado por Carrió y Fayt o la postura asumida por 
se presentan como fundamento de la decisión, sino opinión de los jueces que los suscribieron. En lo que sigue, el trabajo señala las repercusiones favorables que tuvo el fallo en los comentaristas de la época y se concentra en la aplicación del precedente en casos ulteriores, tanto por la propia Corte como por tribunales inferiores.

\section{9. “'Fiorentino' y el derecho 'nocturnal': Una interpretación en clave de justicia transicional". Margarita Maxit y Juan F. González Bertomeu}

Las garantías en el proceso penal constituyen el último "foco jurisprudencial" analizado en la La Corte Genaro Carrió. La tarea queda en manos de Margarita Maxit y Juan F. González Bertomeu, quienes las abordan a partir del caso "Fiorentino".

Los autores, lejos de limitarse a analizar los aspectos normativos de la decisión, indagan sobre su importancia específica en el contexto de la transición democrática, para finalmente abarcar los distintos fundamentos y el alcance de la regla de exclusión, y las implicaciones negativas que podría acarrear abandonar el criterio sentado en "Fiorentino".

$\mathrm{Al}$ igual que en los trabajos anteriores, Maxit y González Bertomeu, comienzan por presentar los hechos del caso: en 1981 un joven es detenido por la policía cuando ingresaba con su novia en el edificio en el que vivía con sus padres. Al ser "interrogado" el joven habría reconocido "espontáneamente" poseer marihuana en su departamento y "autorizado" el registro domiciliario. Los padres, que se encontraban en la cocina al momento del ingreso al domicilio, no se habrían opuesto "de viva voz".

Si bien la defensa cuestionó la legalidad del procedimiento, por ser contrario a la garantía de la inviolabilidad del domicilio, el juez no le hizo lugar por entender que el allanamiento había sido "consentido".

Llevadas las actuaciones ante la Corte, el tribunal se pronunció en una sentencia conformada por un voto mayoritario - suscripto por Caballero,

solo lo que acontece en el fuero íntimo de las personas sino también las conductas externas de los individuos. 
Fayt y Belluscio- - y un voto concurrente - firmado por Petracchí-. Ambos votos coincidieron en identificar los puntos a resolver y el tipo de respuesta que dieron. Mas difirieron en el nivel argumentativo que desarrollaron.

Siguiendo la postura de los firmantes, los autores identifican dos cuestiones a resolver:

- Si la vía recursiva ante la Corte podía abrirse para analizar la validez del allanamiento o si, por el contrario, se trataba de una cuestión procesal ajena al recurso extraordinario federal, como había sostenido el tribunal en "Gullo". ${ }^{17}$

- Si debía desecharse la prueba obtenida en forma ilegal o podía ser considerada en el pleito a pesar de su ilegalidad de origen.

Como muestran Maxit y González Bertomeu, en "Fiorentino" el tribunal decidió expresamente separarse del precedente "Gullo" por entender que la sustancia del planteo lleva a determinar el alcance de la garantía constitucional de la inviolabilidad del domicilio. En este marco, el tribunal efectuó una interpretación robusta en la que dio contenido a la garantía de la inviolabilidad del domicilio y enfatizó que esta debe ser respetada sin condicionamientos ni excepciones. Al hacerlo —agregan Maxit y González Bertomeu - no solo resolvió sobre la admisibilidad del recurso extraordinario, sino que "federalizó" la garantía lo cual — concluyen — tiene implicaciones normativas y remediales significativas.

Una vez resuelta la invalidez del registro, la Corte resolvió también la invalidez del secuestro practicado en esas circunstancias, siguiendo los precedentes sentados por el tribunal en "Charles Hnos" y "Montenegro".

De la decisión del caso, Maxit y González Bertomeu extraen dos reglas:

- La validez de un proceso de allanamiento constituye cuestión federal suficiente, pues gira en torno a la definición del alcance de la garantía de inviolabilidad del domicilio.

17 Esta cuestión incluye una tercera: definir cuál es el alcance de la garantía constitucional de la inviolabilidad del domicilio. 
- Un procedimiento de allanamiento es inválido, y debe desecharse la prueba que de él se obtenga, si aquél no cuenta con autorización judicial y no se da alguno de los supuestos que la exceptúa.

Destacan, no obstante, que en el fallo quedó abierta la cuestión respecto de cuáles son estos supuestos, mas allá de los contemplados en la ley procesal. El consentimiento libre - agregan- podría ser tomado como uno de ellos, lo que parece implícitamente aceptado en el voto mayoritario en "Fiorentino" y expresamente en el voto de Petracchi.

En lo que respecta a la relevancia que cabe asignar al caso en la reinstauración de la democracia, Maxit y González Bertomeu destacan que se trata de una decisión a favor de la protección de esta garantía en un momento del país en el que tenía importantes consecuencias. Recuerdan que la ilegalidad con la que se manejaron las fuerzas de seguridad durante la dictadura no se limitó a quienes fueron víctimas del terrorismo de Estado, sino que se hizo extensiva a la criminalidad ordinaria. De allí que, sostengan los autores, el fallo puede ser considerado como una herramienta de justicia transicional al poner en cuestionamiento la validez del accionar estatal desde el prisma constitucional. Desde un plano simbólico y expresivo, concluyen, el fallo marcó un quiebre con el pasado para abrazar la nueva legalidad.

En lo que sigue, Maxit y González Bertomeu pasan revista a los casos resueltos por el tribunal con posterioridad a "Fiorentino". Muestran que, si bien las cuestiones constitucionales vinculadas a la inviolabilidad del domicilio fueron cambiando, el tribunal habilitó su intervención y resolvió en consecuencia, en los casos en que estuvo en juego la observancia de la doctrina sentada en "Fiorentino".

Finalmente, en la última parte del trabajo, los autores amplían el ámbito de análisis para indagar el valor de la regla de exclusión en un nivel más general y explorar las consecuencias de adoptar algunas de sus posibles justificaciones. 


\section{A modo de cierre}

El proyecto "La Corte y sus presidencias" inaugura un nuevo ángulo de análisis de la producción de la Corte Suprema de Justicia argentina que toma como criterio de clasificación sus propias presidencias. Dicho enfoque se caracteriza por la relevancia que atribuye al contexto político y social en el que actúa el tribunal y por ampliar la mirada para abarcar tanto la producción jurisdiccional como no jurisdiccional.

La Corte Genaro Carrió se presenta como el primer corte temporal en el que se aplican las herramientas metodológicas desarrolladas en aquella investigación.

El resultado es una obra colectiva y plural, entretejida entre directores, editores y autores, en la que cada texto independiente se enlaza con los demás a partir de una línea argumentativa común, centrada en el momento histórico en el que le toca actuar al tribunal y la preocupación por sentar las bases de una cultura del argumento para una sociedad democrática.

\section{Bibliografía}

Abásolo, E. (2013). La Corte Suprema durante el régimen peronista (19471983). En Santiago, A. (dir.), Historia de la Corte Suprema Argentina (pp. 481-525). Buenos Aires: Marcial Pons.

Benedetti, M. (2019a). Una reconstrucción (no jurisdiccional) de la Corte presidida por Genaro Carrió. Tras los rastros de sus acordadas y resoluciones más significativas. En L. Clérico y P. Gaido (eds.), La Corte Genaro Carrió (pp. 79-121). Buenos Aires: Ad Hoc.

Benedetti, M. (2019b). Los otros presidentes: designación y duración de la presidencia de la Corte Suprema Argentina (1863-2018). En L. Clérico y P. Gaido (eds.), La Corte Genaro Carrió (pp. 259-290). Buenos Aires: Ad Hoc. Castagnola, A. (2020). La trampa de la manipulación judicial: Un análisis histórico de la manipulación política de la Corte Suprema argentina. Revista Uruguaya de Ciencias Políticas, 29(1), 49-79.

Cayuso, S. y Gelli, M. A. (1988). Ruptura de la legitimidad constitucional. La Acordada de la Corte Suprema de justicia de la Nación de 1930. 
La Corte Genaro Carrió: una nueva mirada sobre la producción de la Corte Suprema

Recuperado 29/01/2021 de http://www.derecho.uba.ar/investigacion/ Cuadernos_de_Investigaciones1.pdf

Clérico, L. y Gaido, P. (eds.) (2019). La Corte Genaro Carrió. Buenos Aires: Ad Hoc.

Ely, J. H. (1997). Democracia y desconfianza. Santa Fe de Bogota: Siglo del Hombre Editores.

Federal Judicial Center. Supreme Court of the United States: Chief Justices. Recuperado 29/01/2021 en https://www.fjc.gov/history/courts/ supreme-court-united-states-chief-justices

Filippini, L. (2019). Justicia militar; justicia federal. En L. Clérico y P. Gaido (eds.), La Corte Genaro Carrió (pp. 123-157). Buenos Aires: Ad Hoc.

Garay, A. (2019). Genaro Carrio. En L. Clérico y P. Gaido, La Corte Genaro Carrió (pp. 63-77). Buenos Aires: Ad Hoc.

García Mansilla, M. (2020). En Contra de otro 'Court-Packing Plan' Criollo. Recuperado 29/01/2021 de http://endisidencia.com/2020/06/ en-contra-de-otro-court-packing-plan-criollo/.

Helmke, G. (2017). Judicial Manipulation in Latin America. Recuperado 29/01/2021 de https://www.gretchenhelmke.com/ uploads/7/0/3/2/70329843/judicial_manipulation_helmke.pdf.

Nino, C. S. (1983). Una nueva estrategia para el tratamiento de las normas de facto. La Ley, 1983-D, 935.

Nino, C. S. (1985) La validez del derecho. Buenos Aires: Astrea.

Nino, C. (1992). Fundamentos de derecho constitucional. Buenos Aires: Astrea.

Nino, C. S. (2015). Juicio al mal absoluto. ¿Hasta dónde debe llegar la justicia retroactiva en casos de violaciones masivas de los derechos humanos? Buenos Aires: Siglo veintiuno.

Pellet Lastra, A. (2001). Historia política de la Corte (1930-1990). Buenos Aires: Ad-Hoc.

Pérez- Liñan, A. y Castagnola, A. (2017). Los legados de la inestabilidad judicial en América Latina. Perspectivas Revista de Ciencias Sociales. 2(4), 52-73.

Petracchi, E. (1988). La democracia y el Poder Judicial. Lecciones y Ensayos, 49, 301-309.

Santiago (h), A. (dir.) (2013-2014). Historia de la Corte Suprema Argentina. Buenos Aires: Marcial Pons. 
Vallefín, C. (2015). La presidencia de la Corte Suprema de Justicia de la Nación en la República Argentina (comentario desde la perspectiva de su par de los Estados Unidos de Norteamérica). En M. Carbonell Sánchez, H. Fix Fierro, y D. Valadés (coords.), Estado constitucional, derechos humanos, justicia y vida universitaria (pp. 563-573) México: UNAM.

\section{Acordadas}

Año 1930 - CSJN, Fallos 158:290.

Año 1943 - CSJN, Fallos 196:5.

\section{Fallos}

"Abal" - CSJN, Fallos 248:291.

"Aramayo" - CSJN, Fallos 306:72.

"Arenzón" - CSJN, Fallos 306:400.

"Bignone" - CSJN, Fallos 306:655.

"Bosch" - CSJN, Fallos 306:134.

"Brieva" - CSJN, Fallos 306:1644.

"Buzzetti Thomson" - CSJN, Fallos 306:1421.

"Charles Hnos" - CSJN, Fallos 42:157.

“Competencia No 40" - CSJN, Fallos 306:303.

"Dufourq" - CSJN, Fallos 306:174.

"Fiorentino" - CSJN, Fallos 306: 1752.

"Fiscalía Nacional de Investigaciones Administrativas" - CSJN, Fallos 306:271.

"Gary" - CSJN, Fallos 306:035.

"Gauna" - CSJN, Fallos 306:63.

$\forall \quad$ “Giusti”- CSJN, Fallos 306:435.

"Gullo" - CSJN, Fallos 301:676.

"Montenegro" - CSJN, Fallos 303:1938

"Ponzetti de Balbín" - CSJN, Fallos 306:1892.

"Sagasta" - CSJN, Fallos 241-50.

"Videla" - CSJN, Fallos 306:2101. 